\title{
Predicting Fatigue for Isolated Joints While Wearing an Extra- vehicular Mobility Unit (EMU)
}

\author{
James C. Maida \\ NASA JSC \\ L. Javier. Gonzalez \\ Johnson Engineering \\ Sudhakar Rajulu \\ NASA Space Biomedical Institute (NSBRI) \\ Erica Miles \\ Lockheed martin
}

Copyright $\odot 2000$ Society of Automotive Engineers, Inc.

\begin{abstract}
To work outside a space craft, humans must wear a protective suit. The required suit pressurization creates additional resistance for the wearer while performing work. How much does the suit effect work and fatigue? To answer these questions, dynamic torque was collected for the shoulder, elbow and wrist for six subjects in an Extra-vehicular Mobility Unit (EMU). In order to quantify fatigue, the subjects were to exert maximum voluntary torque for five minutes or until their maximum fell below $50 \%$ of their initial maximum for three consecutive repetitions. Using the collected torque and time data, logarithmic based functions were derived to estimate torque decay to within an absolute error of $20 \%$. These results will be used in the development of a generalized tool for prediction of maximum available torque over time for humans using the current EMU.
\end{abstract}

\section{INTRODUCTION}

Complex and labor-intensive extra-vehicular activities (EVA) will be required for construction and maintenance of the International Space Station (ISS). The number and frequency of these activities will also increase. Issues pertaining to human performance while wearing a space suit (EMU) need to be better understood, in order to predict performance for better mission planning, as well as, to improve tools and suits. This project was conducted to investigate how much a pressurized Extravehicular Mobility Unit (EMU) affects human upper body joint strength and fatigue and to predict maximum strength over time (time to fatigue) and total work from computer models based on the data collected.

\section{DATA COLLECTION}

METHOD - Three males and three females with extensive space suit experience were measured both unsuited and suited. Testing was conducted at the Precision Air Bearing Facility (PABF) at Johnson Space Center. This facility allows for rapid configuration changes involving heavy test equipment, such as the support system required for suited subjects. A LIDO strength dynamometer was used to measure joint torque in isokinetic concentric/concentric mode at a constant velocity of 1.02 radians per second $(60 \mathrm{deg} / \mathrm{sec})$. All testing was performed on the subject's right side (five subjects were right handed, one left handed). Torque, angle data were displayed real-time and stored using a LabVIEW data acquisition system.

EVA Suit - During testing, a floor sled was used to relieve the test subject of the mass of the suit (approximately 118 to $123 \mathrm{~kg}$ ). Subjects were in the standing position for all cases. The suit, an Extravehicular Mobility Unit (EMU), was pressurized to $29.6 \mathrm{kPa}$ which is the approximate standard operating pressure when used on orbit. Subjects were closely monitored by a specialized suit technician.

Dynamometer - Joint strength and fatigue measurements were made using a LIDO Multi-Joint II testing unit (Loredan Biomedical, Inc., West Sacramento, CA). The LIDO Multi-Joint II is an integrated system consisting of a dynamometer connected to a personal computer. The system has a series of attachments that enable strength measurements of the various joints of the human body. The system allows setting of experimentally defined exercise parameters such as: velocity, range of motion (ROM), and torque limits. A unique feature of the LIDO 
is that it incorporates a gravity compensation feature that takes into account the one-gravity (1G) artifact throughout the ROM. This capability is used to remove the effect of the weight of the LIDO attachment, EMU suit segment, and the subject's limbs from the measured torques and forces, providing the subject's actual effort.

Figure 1. Example of Test Configuration

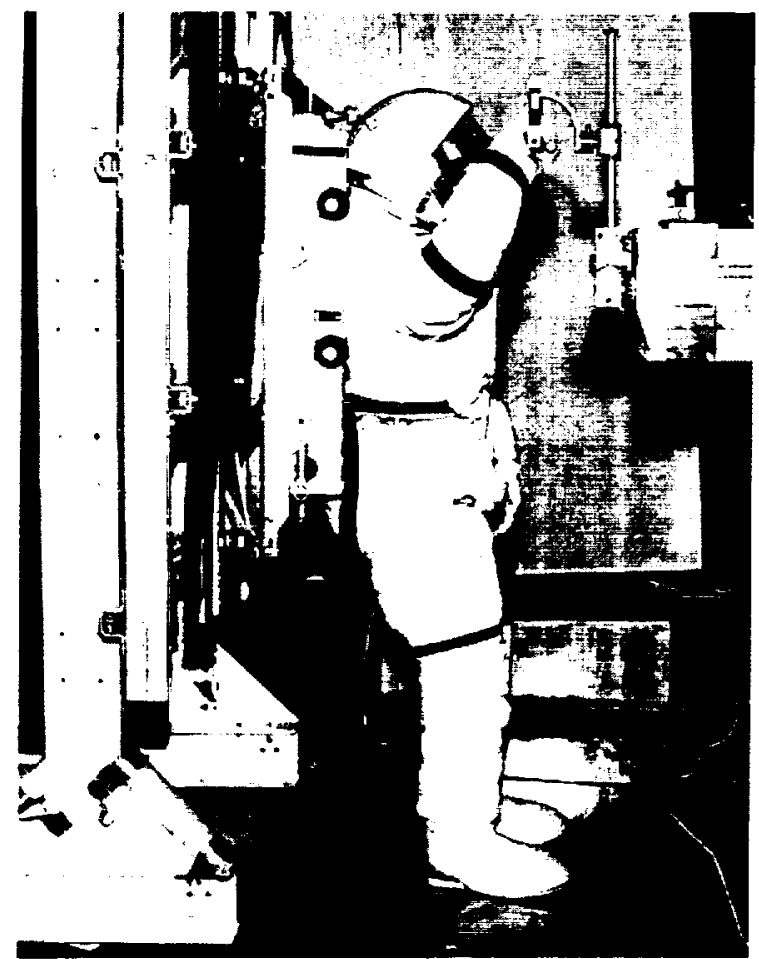

PROCEDURE - Since upper-body movements (e.g., locomotion using hand rails, using tools to repair objects, and moving large masses) are integral to most ExtraVehicular Activity (EVA) operations, emphasis was placed on testing strength and fatigue for the major joints of the upper body. The five isolated isokinetic joint motions that were included were: (1) shoulder flexion/extension, (2) shoulder abduction/adduction, (3) shoulder internal/external rotation, (4) elbow flexion/extension, and (5) wrist flexion/extension. All of the testing was performed on the subject's right side with the subject secured in an upright (standing) posture.

Measuring Maximum Voluntary Torque (MVT) - To determine MVT, the subject rotated the LIDO attachment back and forth using maximum exertion at a prescribed angular velocity of $1.05 \mathrm{rad} / \mathrm{s}$ throughout the ROM for three non-stop repetitions. Because of time constraints imposed by suit availability only one angular velocity was used. The variation between the three torque curves was then computed using the LIDO computer. A variation of more than ten percent was used as a measure to indicate that the subject was not using maximal exertion during the strength test. If the variation was less than ten percent, the subject's MVT for both directions of motion was computed by averaging the three peak torque values for each direction.
Measuring Fatigue - For the fatigue tests, the subject rotated the LIDO attachment repeatedly at the prescribed angular velocity of $1.05 \mathrm{rad} / \mathrm{s}$ throughout the entire ROM using the target level of exertion $(100 \%$ or $80 \%$ of the MVT) without stopping. The subject was allowed to continue the test until the torque output in both directions dropped below the defined fatigue index value $(50 \%$ of the MVT) for three consecutive repetitions. If the subject did not reach fatigue after five minutes, the test was stopped. A five-minute rest period was allowed between joint measurements. This procedure was used for both the unsuited and suited tests. Figure 2 is the test protocol as pseudo-code.

\section{Figure 2. Test Protocol}

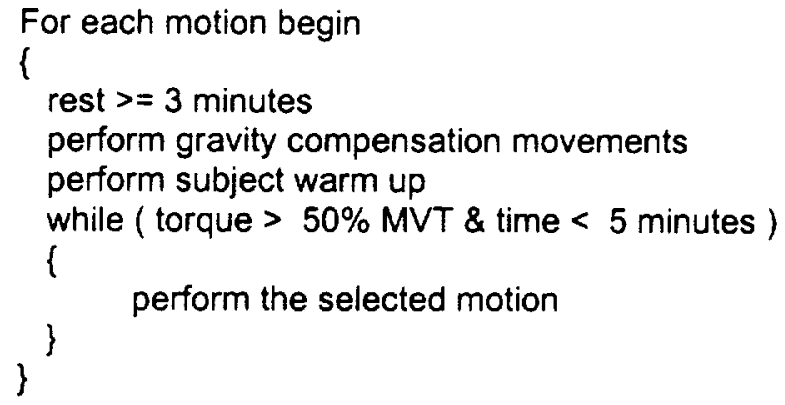

\section{DATA ANALYSIS AND PREDICTION FUNCTIONS}

DATA ANALYSIS - The effects of an EMU on human performance were investigated. The unit is worn by astronauts while working outside of their space craft in low earth orbit. Isolated isokinetic joint torques of three female and three male subjects (all experienced users of the suit in 1G) were measured while working at $100 \%$ and $80 \%$ of their MVT. It was found that the average decrease in the total amount of work done when the subjects were wearing the EMU was $48 \%$ and $41 \%$ while working at $100 \%$ and $80 \%$ MVT, respectively. There is a clear relationship between the MVT and the time and amount of work done until fatigue. In general the stronger joints took longer to fatigue and did more work than the weaker joints. However, it is not clear which joints are most affected by the EMU suit in terms of the amount of work done. The average amount of total work done increased by $5.2 \%$ and $20.4 \%$ for the unsuited and suited cases, respectively, when the subject went from working at $100 \%$ to $80 \% \mathrm{MVT}$. Also, the average time to fatigue increased by $9.2 \%$ and $25.6 \%$ for the unsuited and suited cases, respectively, when the subjects went from working at $100 \%$ to $80 \% \mathrm{MVT}$.

TORQUE PREDICTION - In addition to the data analysis above, it was also found that the experimentally measured torque decay could be predicted by a logarithmic equation. The average error in the predictions was found to be $\pm 9.2 \%$ and $\pm 9.5 \%$ for the unsuited and suited subject, respectively, working at $100 \%$ MVT, and $\pm 11.3 \%$ and $\pm 9.4 \%$ for the unsuited and suited subject. respectively, working at $80 \% \mathrm{MVT}$. 
Figure 3. Actual and Predicted for Wrist Extension at $100 \%$ MVT (lower plot is suited condition)

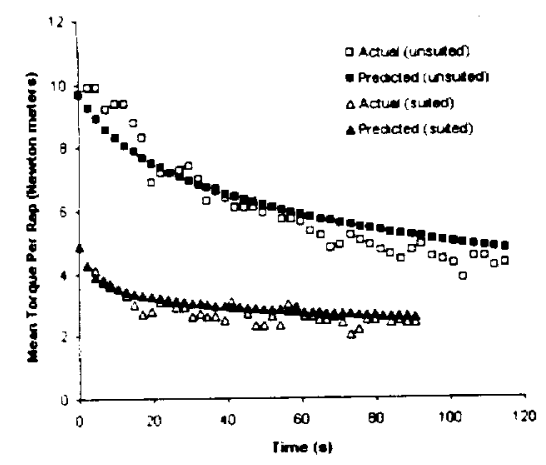

Actual and predicted torque for subject 2 during wrist exterision at $100 \%$ MVT

A MATLAB routine was written to predict the torque surface as a function of joint angle and time given the peak torque in the first repetition of a repetitive task. Alternatively, the MVT of the subject could also be used as the peak torque in the first repetition of a repetitive task. The method basically uses to normalized curves to generate the torque surface, these two curves are: (1) the curve representing the torque decay as a function time, and (2) the curve representing the torque as a function of joint angle for the first repetition of a repetitive task. The average error in the predictions was found to be $\pm 12.2 \%$ and $\pm 16.1 \%$ for the unsuited and suited subject, respectively, working at $100 \% \mathrm{MVT}$, and \pm $13.5 \%$ and $\pm 18.7 \%$ for the unsuited and suited subject, respectively, working at $80 \%$ MVT. Also, a compact method of transporting the EMU Fatigue Data was a.so developed in MATLAB. This method of compactly transporting the data involves representing the data by two curves rather than approximately 15,000 data points. The method used to store the data in a compact form is a variation of the method described above to develop the torque surface predictions.

Figure 4. Torque Surface - Torque vs Joint Angle Over Time

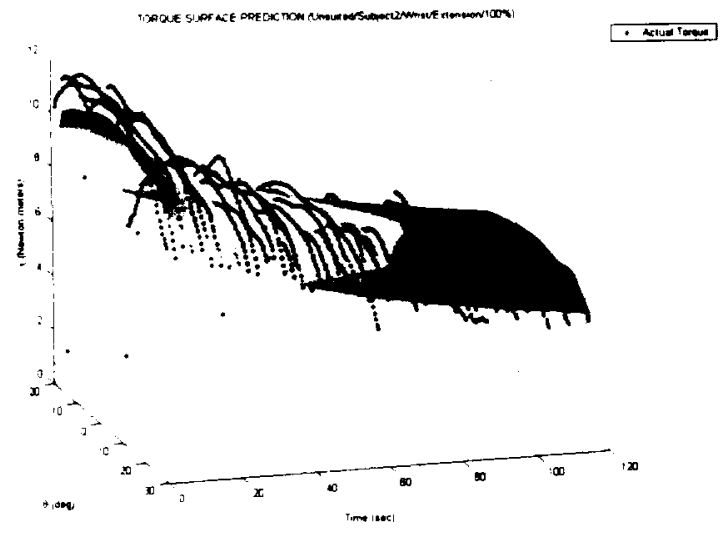

\section{CONCLUSION}

RESULTS - The effects of an EMU on isolated joint torque were measured. Using six subjects, both suited and unsuited, isolated isokinetic joint torques were measured with the subjects working at $100 \%$ and $80 \%$ of their MVT, using $50 \%$ of the MVT as the fatigue index. It was found, on average, that the EMU reduced total work by about $50 \%$, and the larger the MVT, the longer the time to fatigue and the greater amount of work done. It was not clear which joint the EMU suit affects most. The amount of work and time to fatigue improved the most for the suited case, when the subject started at $80 \%$ MVT. It was also found that the experimentally measured torque decay trend could be predicted by a logarithmic equation. MATLAB routines were also written to predict the torque surface as a function of joint angle and time given the peak torque in the first repetition of a repetitive task, and to transport the EMU Fatigue Data in a compact form

FUTURE WORK - The fatigue measurements on the upper body were only done at one joint velocity. Future work must include the investigation of the velocity effects on fatigue for the upper body joints. Also, these experiments must also be repeated for all the major joints of the lower body. Future work also includes the incorporation of these results into a human threedimensional dynamic model.

\section{ACKNOWLEDGMENTS}

Thanks to Abhilash Pandya, Wayne State University, for his ideas and work at the beginning of this project.

This project was funded under NASA grant (96-HEDS01).

\section{CONTACT}

James.Maida@jsc.nasa.gov

Luis.J.Gonzalez1@jsc.nasa.gov

Sudhakar.Rajulu@jsc.nasa.gov 
